Epilogue: Tribute to Sandro and Erika

Fl. Medit. 31 (Special Issue): 521-544

https://doi.org/10.7320/FlMedit31SI.521

Version of Record published online on 6 January 2022

\title{
Cesare Lasen
}

\section{Biodiversité végétale, valeurs naturelles et sauvegarde du paysage dans le domaine dolomitique}

\begin{abstract}
Lasen, C.: Biodiversité végétale, valeurs naturelles et sauvegarde du paysage dans le domaine dolomitique. - Fl. Medit. 31 (Special Issue): 521-544. 2022. — ISSN: 1120-4052 printed, 2240-4538 online.

This contribution summarizes the state of the geobotanical knowledge of the Dolomite area starting with the excursions made in the early 1970s with Erika and Sandro Pignatti. The references are limited to the main titles and a meaningful increase in knowledge has been detected, especially at floristic level, also with the discovery of new taxa. The Dolomites were recognized as a World Heritage Site in 2009 by UNESCO on the basis of geological and landscape criteria. The conservation and protection of the Dolomite landscape therefore assumes priority in a historical period in which the natural resources of the planet are increasingly threatened by interventions and projects based on further exploitation initiatives, called "enhancement" for touristic purposes. The article refers to the centrality of geobotanical knowledge and the priority to be attributed to biodiversity, also considering the current evolutionary dynamics. Adequate protection of naturalistic values, as well as landscape values, should be guaranteed by the existence of a large network of national, regional protected areas and/or belonging to the Natura 2000 network.
\end{abstract}

Key words: Dolomites, plant biodiversity, protected areas, nature conservation, evolution dynamics.

\section{Introduction}

Les excursions dans le territoire dolomitique avec Erika et Sandro Pignatti, pendant les années '70-80, ont profondément marqué la mémoire et le coeur. Les exemplaires de l'herbier, aujourd'hui conservés au Musée Naturalistique du Parco Nazionale Dolomiti Bellunesi, en sont le témoignage. C'est justement avec eux et avec le maître Armando Scopel qu'on a publié (Lasen \& al. 1977) la "Guida Botanica des Dolomiti di Feltre e Belluno" dans la précise intention de pousser à la réalisation, au niveau de données scientifiques, du Parco Nazionale, depuis longtemps imaginé et désiré. Cet espoir s'est concrétisé une douzaine d'années plus tard par le D.M. du 20-04-1990 qui en établissait les limites avec la dénomination de Parco Nazionale delle Dolomiti Bellunesi et avec il DPR du 12-07-1993 qui instituait l'Ente Parco siégé à Feltre et dont je fus nommé Président. 
Après avoir résumé les éléments d'importance cardinale, même pour ceux qui ne connaissent pas ce territoire, en rajoutant les connaissances sur la flore et la faune, cette prémisse est nécessaire pour comprendre le point de vue de cette contribution avec laquelle on veut concentrer l'attention sur le thème, toujours actuel et souvent mal compris, de la conservation de la Nature dans le sens plus exhaustif du terme, conjuguant les valeurs scientifiques, par chance, encore solides et sans la nécessité de contrainte pour être mises en évidence, avec le réalisme de la gestion qui impose des solutions d'équilibre et de courage, imprévues et non passives. Je suis sûr que Sandro, collègue dans la première Consulta Nazionale pour les Aree Protette, présidée par le Maître Gianluigi Ceruti, l'un des pères de la loi-cadre 394/91, partagera ce choix qui s'adresse, ici, à un milieu territorial local, encore que les Dolomites soient célèbres dans tous les continents. Mais ce choix veut se projeter sur un scénario global qui voit la planète en grave souffrance. Même le Pape François l'a mis en évidence avec un appel plein de tristesse dans l'encyclique "Laudato sì", publiée le 18 juin 2015.

\section{L'état des connaissances}

Le territoire dolomitique a fait l'objet (et encore actuellement) de nombreuses publications sur l'aspect naturel, en particulier géologique et du paysage. Au niveau botanique, en ce qui concerne les plantes vasculaires, les contributions scientifiques sont, elles aussi, importantes pour le nombre et la qualité. Pour des raisons d'espace, évidemment, on se limitera à considérer les textes les plus récents et moins localisés, vu que quelques-uns de ces textes contiennent de précises références bibliographiques qui permettent de puiser aux sources de l'exploration de la flore.

La panoramique la plus exhaustive sur la flore et la végétation du territoire dolomitique, c'est précisément celle publiée, en trois volumes, entre 2014 et 2017 par Erika et Sandro Pignatti.

Ils ont commencé à étudier les Dolomites vers la fin des années '60 et début des années ' 70 . Nombre de données et de reliefs remontent à cette période, entretemps les connaissances de la flore ont évolué. Essentiellement la position écologique et sur la végétation n'a pas changé, tandis que la syntaxonomie ressent de différentes approches et elle rend difficiles les comparaisons. Pendant cette période on a eu de nombreuses occasions de collaboration qui ont abouti, même, à quelques publications. Outre la "Guida Botanica" (Lasen \& al. 1977), ci-dessus mentionnée et rapidement épuisée, qui a représenté un fort élan pour la promotion de l'institution du Parco Nazionale, il faut considérer le volume de Studia Geobotanica ( ${ }^{\circ} 3,1984 a$, soigné par les deux), dédié exprès, avec de différentes contributions, à la flore et à la végétation des Vette Feltrine, mais qui, en réalité, rapporte aussi des reliefs effectués sur les montagnes aux alentours de Belluno. Toujours à propos du milieu dolomitique, ensuite on a publié une étude sur les groupements à Matteuccia (Lasen \& Pignatti 1992) et en 2002 (Lasen \& Pignatti) une tentative pour typer les sapinières. A partir de 1960 (sur la végétation casmophile à Physoplexis comosa et Asplenium seelosii), il y a déjà de nombreuses contributions sur la végétation avec la description de nouvelles communautés végétales ou dans le but de les ajouter. Après la "Guida Botanica alla Val di 
Fassa” (Pignatti E. \& Pignatti S. 1974), pendant une excursion de la Società Botanica, Erika et Sandro recommencèrent à étudier la végétation des parois rocheuses en décrivant le Campanuletum morettianae (1978) et, en 1985, le Caricetum rupestris des milieux herbeux-rupestres, fouettés par le vent. En 1981 ils anticipèrent les nouveautés de leurs recherches par la descriptions de nouvelles communautés. En 1983 ils typèrent un Danthonio-Nardetum, écologiquement très clair, même si aujourd'hui il n'est pas reconnu avec ce nom. En 1984 (b) ils s'occupèrent de la syntaxonomie des éboulis de faille et, en 1988 (b) des prairies semi-grasses à Trifolium pratense subsp. nivale, (Knautio-Trifolietum, terme tombé en désuétude, mais lui aussi écologiquement explicite). Ce n'est qu'en 1995 que l'on publiera une première liste des unités végétales. On peut relever l'évolution syntaxonomique, en comparant le prodrome italien (Biondi \& al. 2014), mais il existe des synthèses à niveau européen qu'il faut considérer. Datée 1981 (œuvre de Sandro), très importante au niveau méthodologique, il y a la publication d'un plan des ensembles de végétation pour la Conca Ampezzana. Dommage, par la suite l'idée de relever les ensembles de végétation, pour décrire le paysage, n'a pas eu le succès qu'elle aurait mérité. Les articles du 1988 (Pignatti \& Pignatti 1988a), du 2003 et celui signé par Sandro en 1998 ont une position surtout sur le paysage. Pour la flore, comme l'on sait, il a été, pour longtemps, le promoteur du relèvement cartographique par quadrants et l'on commençait à penser aux archives informatisés (Pignatti \& al. 1966). A partir de la seconde moitié des années '70, grâce aux nombreuses excursions ensemble, on a exploré d'autres domaines et l'on a approfondi les connaissances sur les territoires de particulière valeur naturelle. Avec les pharmaciens Thea Saffaro et Italico Boiti, résidants à Predazzo, on a publié une monographie sur la Val Venegia (Boiti \& al. 1989) après avoir anticipé (Boiti \& al. 1988) une contribution sur les alluvions des torrents glaciaux avec le relèvement sur Caricion maritimae. Aujourd'hui encore, les terrasses sur le fond de la vallée du Travignolo, depuis les sources jusqu'à Malga Venegia, abritent des espèces d'une valeur naturelle extraordinaire telles que Carex microglochin, Carex bicolor, Juncus arcticus, outre à des briophytes, Kobresia simpliciuscula, Juncus triglumis et d'autres. Dans la même vallée, les groupes de saules aussi sont remarquables (Lasen 1989b). Avec Marcello Tomaselli et Italico Boiti on a traité de la végétation des plateaux glacio-karstiques. Avant la reconnaissance formelle des Dolomites comme patrimoine sériel naturel de l'humanité (en 2009, si cfr. Lasen 2012; Viola \& al. 2005), il semblait important de proposer des synthèses de vulgarisation (Lasen 2000, 2001). Une fois fondé le Parco Nazionale des Dolomiti Bellunesi en 1990 (l'Ente de gestion en 1993), il y eut de nouvelles raisons pour approfondir les recherches. La première ceck-list du Parco et des aires limitrophes, avec 1700 espèces, date de 2001 (Argenti \& Lasen). Les mêmes auteurs en 2004 publièrent la ceck-list de toute la province avec la liste rouge (pour le Trentino, elle avait été rédigée par Prosser en 2001) qui fut rajoutée, après, pour la totalité du territoire provincial dans la volumineuse œuvre de Prosser \& al. en 2019. Des ajoutés à la liste rouge du territoire de Belluno ont été publiés en Buffa \& al. (2016). Indépendamment de chaque contribution aux ajoutés, souvent publiée dans la revue Gredleriana pour la Provincia Autonoma de Bolzano, la référence de base reste le «Katalog» (Wilhalm \& al. 2006). Il y a aussi plusieurs contributions publiées pour les territoires du Friuli entre lesquelles l'atlas chorologique de Poldini (2002) qui est 
une référence valable. Les contributions, concernant surtout la végétation, sont également nombreuses. Pour le territoire du Parco Nazionale (Lasen 2004) et pour le territoire provincial (Lasen 2008, dans les "Tesori Naturalistici" qui traite aussi le thème du paysage, 2020a). En ce qui concerne l'autre parc de la province, celui régional des Dolomiti d'Ampezzo, les études publiées sont remarquables, à partir des recherches bien localisées sur quelque biotope (Tomaselli \& al. 2006; Da Pozzo \& Lasen 2010), à d'autres notes de synthèse (Lasen \& Da Pozzo 2011; Da Pozzo \& al. 2016), à la découverte d'une rareté, Carex maritima (Lasen \& Da Pozzo 2017). Il serait interminable de citer toutes les contributions intéressantes soit sur la flore, soit sur la végétation ou les paysages (les nombreuses brochures des cours de géographie organisés par la Fondazione Angelini rentrent dans cette catégorie) concernant chaque site, biotope, vallée.On peut citer, par exemple, une note de synthèse sur les Dolomites de Sinistra Piave (Lasen 2009) et un volume dédié à la Forêt de Somadida (Del Favero \& Lasen 2016). Après l'événement de Vaia, on a publié plusieurs contributions (Lasen 2020b), mais celles à position écologique et de la végétation sont limitées et parfois, encore en cours d'imprimerie. Grâce aux banques des données de la flore (Musée de Rovereto et de Bolzano), les niveaux de connaissance pour les provinces autonomes sont avancés. Pour Belluno aussi, la couverture est importante, malgré l'absence d'organisme institutionnel préposé, mais grâce au travail des volontaires. Il en va de même pour le domaine dolomitique plus oriental, sachant que pour le FVG un nouveau atlas, fondé sur des quadrants à l'échelle 1:25.000 au lieu de 1:50.000 (Martini ex verbis), devrait être publié. Pour les données sur la végétation, la situation est plus complexe. Il y a de nombreuses contributions à l'échelle locale. La synthèse des Pignatti est certainement remarquable à l'échelle écologique générale, tandis qu'il manque encore une vraie et propre liste de chaque phytocénose qui tienne edonnée.sn compte les acquisitions syntaxonomiques les plus récentes. Le paysage a été l'objet de plusieurs études, mais les changements en action nous rappellent que tous les détails ne sont pas éclaircis et, en particulier, les successions écologiques du post-Vaia démontrent que nos connaissances sont encore limitées et incomplètes.

\section{Valeurs naturelles et du paysage}

Pour ce qui concerne la flore, il est relativement simple de définir qualitativement les indicateurs qui contribuent à tracer un tableau de valeurs. Le composant de la quantité, fondé essentiellement sur la richesse des espèces (par exemple la flore vasculaire qui est plus facile à évaluer), fournit moins d'informations puisque un élément de dérangement ou causant quand même hétérogénéité (un sentier qui traverse le bois, des passages ou lieux de repos des animaux sauvages) provoque une augmentation des espèces pour la plupart banales et qui ne représentent sûrement pas un indice de qualité. Dans la littérature on trouve nombre d'études et de modèles qui ont cherché à offrir un tableau avec des points attribués à de différents paramètres dont la somme permet, enfin, de trouver des valeurs utilisables pour des confrontations, soit dans le champ de l'application (pâturages, forêts), soit pour des évaluations d'impact. Parmi les premières études, suivant plus une école naturelle plutôt que d'écologie appliquée, 
on rappelle celle de Poldini \& Pertot (1991) sur le Karst de Trieste. L'utilisation d'organismes comme bio-indicateurs du milieu a été examinée avec beaucoup de contributions et, entre autres, on rappelle le volume par soin de Gasparo \& Zappa, en 1994, toujours publié à Trieste. Ce thème a vu fleurir des articles et des rencontres, précisément dans les années à cheval sur les deux siècles et, entres autres, on cite Tomaselli \& Gualmini (2000) qui ont mis au point le problème au niveau, surtout, des communautés végétales. Pour ses articulations et ses variétés des thèmes le volume soigné par Pirola (2000) reste intéressant.

Le travail de Melzer (1999) sur le macrophytes aquatiques que l'on considère comme des indicateurs du niveau de nourrissants, est historique et assez utilisé (Lasen \& Scariot 2007). Pour les cours fluviaux, assez connu est le travail de Lippert \& al. (1995), d'où il apparaissait la remarquable qualité du milieu des biocénoses le long du fleuve Tagliamento. Sur les indicateurs de valeur dans les différents types forestiers, on rappelle une approche, non analytique et assez pragmatique, dans le volume de Del Favero \& al. (2000), le dernier de la série publiée sur les types forestiers du Veneto et certainement encore utile pour la région dolomitique. Bonomi \& Buffa (2000) ont fournit des indications sur l'évaluation de la qualité de la végétation dans les aires de tourbière. A ce propos, aujourd'hui, il serait plus facile d'individualiser les indicateurs les plus idoines, après la publication des listes rouges nationales, régionales et provinciales. Scotton \& al. (1996) et Rodaro \& al. (1998) ont travaillé surtout à l'attribution d'une valeur agronomique aux pâturages (dans les deux cas, dans l'Ampezzano). Ferrari \& al. (2000) proposent une approche quantitative pour évaluer la qualité naturelle et la diversité de la végétation. Comparable, avec l'application à toute la Province de Varese sur une base cartographique, c'est la proposition de Cerabolini \& al. (2007). Toujours pendant les mêmes années (Mazzocchi \& al. 1999), c'est l'ENEA qui publie une étude où l'on évalue la qualité ambiante, en intégrant des données sur la flore et la végétation et cela est bien significatif. En considérant les données dont on dispose, pour la plupart des cas et, certes dans le domaine dolomitique, il serait intéressant de reformuler une vision intégrée qui pourrait fournir des résultats utiles à la prévention des projets d'infrastructures, en multiplication, dans la prévision de grands évènements sportifs. Indépendamment de l'importance quantitative que l'on décidera d'y attribuer, il faut tenir dans la plus grande considération les paramètres suivants:

- Rareté et insertion dans les listes rouges (avec les différents niveaux de menace);

- Importance locale d'espèces et de communautés végétales situées dans des stations séparées, à la limite de l'aire (extra-zonales au niveau de phytocénose);

- Observations récentes relatives à des situations de déclin (réduction de la population et/ou fragmentation de l'habitat);

- Présence d'endémismes de différents niveaux ou d'autres chorotypes d'intérêt phytogéographique

- Situations objectivement de péril, causées par de fortes possibilités que l'espèce et surtout l'habitat souffrent à cause du changement climatique et du dynamisme évolutif défavorable;

- Valeurs éventuelles de caractère historique-ethnographique. 


\section{Récentes acquisitions sur la flore et la végétation}

Le territoire dolomitique s'étend sur des régions et des provinces autonomes, ayant chacune une banque des données qu'on pourrait définir institutionnelle (aux musées de Rovereto et Bolzano), exception faite pour le Veneto où des spécialistes individuels sont en activité, souvent sur base provinciale. En Friuli, on faisait référence à l'Université de Trieste, mais, à présent, on attend la publication d'un nouveau atlas par soin de Fabrizio Martini. Le classement aux archives des données de la végétation est plus compliqué, même si, en général, auprès des musées déjà cités, une banque bibliographique est disponible. Les données, donc, résultent pour la plupart subdivisées au niveau géographique. Comme déjà dans La Flora d'Italia, provocant aussi des critiques de la part de groupes qui se sont vus dépassés, seulement Pignatti a publié une monographie sur le territoire dolomitique, indépendamment des questions administratives. Dans ce paragraphe, d'autre part, on veut tout simplement souligner le progrès des connaissances, pleinement développé, pendant les dernières décennies. Il est vrai que les endémismes, reconnus pour les Dolomites, ne sont pas nombreux, et cela a pesé sur la reconnaissance ratée des critères IX et X pour l'insertion dans la liste du patrimoine de l'humanité par UNESCO. Mais, bien sûr, il faut souligner que la richesse globale, en termes de flore est plus que remarquable par la présence d'espèces rares, menacées, situées aux marges de l'aire et qui ne sont pas, d'autre part, exclusives. Celui-là c'est seulement l'un des critères. Il ne s'agit pas de nombres, mais de la qualité et des valeurs phytogéographiques, bien mis en évidence dans les nombreux travaux publiés. Un exemple: la richesse de la flore est en général plus grande dans les secteurs extérieurs (ésodolomitiques) plutôt que dans ceux intérieurs (endodolomitiques à climat continental). Les études ajoutées (et c'est seulement un exemple) sur le territoire du Parco delle Dolomiti d'Ampezzo (œuvres citées) semblent démentir, au moins au niveau de la quantité, ce présumé, né sur la présence du nombre d'espèces dans les différents quadrants de la Cartografia Floristica dell'Europa Centrale. En revenant aux endémismes (inclus quelques-uns avec peu de stations extérieures au territoire, il vaut la peine de les rappeler: Sempervivum dolomiticum, Saxifraga facchinii, Saxifraga depressa, Draba dolomitica, Rhizobotrya alpina, Campanula morettiana, Prymula tyrolensis, Gentiana brentae (la découverte la plus récente, Prosser \& Bertolli 2008), Nigritella buschmanniae et Festuca austrodolomitica.

Evidemment on ne considère pas les espèces de genre apomictique, Alchemilla, Hieracium, Rubus, Taraxacum pour lesquels il faudrait formuler d'autres types d'observations. En tout cas, leur récolte systématique et les révisions spécialisées prouvent que l'échantillonnage n'est pas complet et que d'autres découvertes sont possibles. Après avoir rappelé que la synthèse de Pignatti n'est pas à considérer exhaustive au niveau d'association et qu'elle reste inspirée à la position écologique classique, il manque encore, au niveau de communautés, une ceck-liste complète, surtout pour les catégories de formes moins naturelles. Cela paraît évident aussi par de récentes listes publiées au niveau européen (Mucina \& al. 2016). Parmi les découvertes les plus étonnantes de ces dernières années, une série d'excursions, en 2014, pour étudier la réserve naturelle de Somadida (gérée par CFS, Ufficio Territoriale pour la Biodiversità de Vittorio Veneto), ont permis de découvrir, dans une zone difficilement accessible, un bois résiduel qu'on 
pourrait définir primordial plutôt que vétuste, avec des mélèzes et des arolles pluriséculaires (non signalés auparavant dans ce territoire). Des recherches successives ont démontré qu'ils étaient âgés de plus de 500 ans. De cette découverte, objectivement aventureuse, on en a traité dans le volume par soin de Del Favero \& Lasen (2016) et dans d'autres articles à caractère scientifique, concernant aussi la faune invertébré. Le bois de Col Nero, déjà clairsemé à cause de l'altitude et sub-rupestre, a subi d'autres espacements forcés à cause des évènements météo extrêmes. Malgré cela, il garde un charme sans pareil qui fait vraiment comprendre la force de la Nature. Au contraire, de nombreuses contributions de récente publication, sont disponibles et elles concernent soit les seuls milieux géographiques, soit des groupements de habitat tels que les forêts, les zones humides, d'intérêt agro-pastoral ou bien des catégories primitives. Ce qui manque, pour le moment, c'est une synthèse sur base régionale. On sait qu'en Alto Adige, on est à l'ouvrage pour des finalités d'application, par un groupe de travail, dans le but d'atteindre cet objectif.

\section{La couverture forestière}

Certes les Dolomites sont célébrées pour leurs paysages, pour les vastes prairies, pour les aiguilles et les crêtes, pour les spectaculaires conoïdes détritiques. Ce sont des milieux merveilleux pour leur noble flore et pour la présence de communautés végétales endémiques et rares. Toutefois, parmi les catégories d'habitat, le rôle prépondérant, non seulement au niveau quantitatif, c'est celui de la couverture forestière. Contrairement à d'autres milieux alpins, depuis n'importe quel sommet dolomitique important, il est toujours possible d'apercevoir la présence des arbres. On ne pourrait pas imaginer un paysage sans les extraordinaires couleurs qui, en automne spécialement, caractérisent les bois latifoliés mixtes ou simplement de conifères. Un court résumé des principaux types et formations contribue à décliner un étonnant tableau riche en variétés, soit en relation à la série d'altitude, soit aux principales différences entre les milieux extérieurs (éso-mésalpiques) et intérieurs (méso-endalpiques) bien plus articulés selon les différents substrats depuis les carbonatés purs jusqu'aux silicatés avec de différentes séries bien exprimées, même si sur une échelle peu détaillée (1:500.000), dans la publication sur les séries végétales (Blasi 2010) dont les textes et les références sur des bases phytosociologiques sont rédigés par de divers auteurs pour les différents territoires régionaux. Depuis les fonds de la vallée extérieurs, encore semblables à ceux préalpins (bois de charmes et chênaies-charmes-Carpinion orientalis et d'autres formations à dominance de Quercus robur dans la vallée du Piave à partir de $250 \mathrm{~m}$ ), jusqu'à la limite supérieure de la forêt et des groupes d'arbres qui, dans les zones internes plus continentales, comprennent des mélèzes (Larix decidua) et des arolles (Pinus cembra) qui s'élèvent jusqu'à 2300-2400 m. Outre les végétations de type azonale (milieux riverains avec des saulaies-Salix sp. pl., parfois avec peuplier-Populus sp., aulnaies d'aulne blanc-Alnetun incanae s.1., avec de modestes coins d'aulne noir-Alnus glutinosa dans des zones marécageuses), ou liées à de particuliers substrats (par exemple des groupes de châtaigneraies à Castanea sativa parfois avec du rouvre-Quercus petraea, toujours bien localisés mais importants pour la biodiversité), on observe une série articulée avec 
d'étonnantes particularités jusqu'à présent approfondies seulement en partie. Et encore, on peut observer des tilleuls-érablières et des érables-frênaies (Tilia platyphyllos, Tilia cordata, Acer pseudoplatanus, Acer platanoides, Fraxinus excelsior, c'est à dire biocénose de Tilio-Acerion s.l.) dans des milieux de ravin ou de versants frais, des bois de Carpinus betulus riches en géophytes (complètement absents en Alto Adige) où le robinier-Chelidonio-Robinietalia, indicateur de bouleversement n'a pas pris le dessus. Sur les versants escarpés des zones inaccessibles, exposés à de fortes variations d'humidité $\mathrm{du}$ sol, toujours dans les secteurs externes, on trouve les formations à Ostrya carpinifolia et Fraxinus ornus, Ostryo-Carpinenion orientalis et Erico-Fraxinion orni avec des groupes peux nombreux mais intéressants de chêne pubescent (Quercus pubescensQuercion pubescenti-petraeae). Dans le paysage dolomitique, toujours sur des sols drainants, capables de supporter l'aridité estivale, sont diffusées les pinèdes, de pin sylvestre (Erico carneae-Pinion sylvestris) pour la plupart, depuis les premiers versants jusqu'à environ 1700-1800 m. d'altitude, mais aussi celles de pin noir (Pinus nigra) dans les secteurs plus orientaux avec plus de précipitations ou d'humidité atmosphérique qui deviennent prédominantes, en alternance avec Fagus sylvatica selon l'évolution du sol, dans les Dolomites du Friuli.

Au-dessus de la zone des bois latifoliés mixtes, où l'on compte aussi des formations secondaires ou des stades sériels avec la prédominance de bouleaux-Betula pendula, de trembles-Populus tremula ou de noisetier-Corylus avellana (expressions de Betulo pendulae-Populetalia tremulae), dans le paysage dolomitique ce sont les hêtraies qui ont une importance remarquable et qui sont dominantes et pures dans les zones préalpines et dans les Dolomites extérieures à climat sub-océanique.

Les hêtraies comprennent de différentes associations de Aremonio-Fagion, depuis celles submontagneuses avec Taxus baccata et/ou Ostrya carpinifolia, jusqu'à celles haut-montagneuses-subalpines avec Polystichum lonchitis, d'autres fougères et abondance d'Acer psueudoplatanus. Bien plus rares sont les hêtraies acidophiles du LuzuloFagion (aux alentours de Gosaldo).

Les hêtraies deviennent mixtes avec le sapin blanc-Abies alba, en formant des bois spectaculaires (sapinières-hêtraie-Abieti-Fagetum) dans l'aire centrale des Dolomites. La sylviculture a favorisé (et on explique ainsi les lacunes de Abies alba dans le territoire de Agordo) le sapin rouge (épicéa, Picea excelsa) répandu partout, mais qui forme d'abord des associations de Abieti-Piceenion ou des Calamagrostio variae-Abietenion sur beaucoup de versants et dans les fonds de la vallée dolomitiques dans le secteur méso-endalpique. Seulement dans le secteur plus à l'intérieur, les bois purs de Picea excelsa prennent leur habit le plus typique et en syntonie avec la végétation potentielle, à partir de la zone montagneuse (900-1000 m.) et ensuite pour entrer en compétition avec les bois de Larici-Pinetum cembrae à partir de 1700-1800 m. Tandis que Pinus cembra (rare à cause du pâturage actuel et d'antan) s'établit seulement dans les secteurs plus continentaux en devenant un très bon indicateur du bioclimat, les bois pionniers de Larix decidua (Rhodothamno-Laricetum) ou plus mûrs aussi, sont répandus dans tout le territoire, même dans les secteurs les plus à l'extérieur, où il manque une vraie et propre zone de conifères et ils suivent en altitude les hêtraies. Il faut considérer que même les arbustes ont un rôle déterminant, non seulement dans le paysage, mais aussi pour la protection du sol. Le symbole dolomitique par excellence peut-être considéré le pin 
couché (Pinus mugo). On rappelle que les bois basophiles de pin couché à Rhododendron hirsutum, en acidifiant le sol, abritent aussi le Rhododendron ferrugineum et ils sont considérés l'habitat prioritaire dans l'Annexe I du Réseau Nature 2000 (avec le code 4070*). Outre les vastes brousses à Pinus mugo, objet de monographies spécifiques, se sont répandues les aulnaies de aune vert-Alnus viridis, Alnetum viridis s.l. (sub-hygrophiles qui couvrent les versants neigeux ou des couloirs exposés aux avalanches sur des terrains avec une bonne rétention hydrique), les étendues acidophiles de rhododendron à Rhododendron ferrugineum, exclusives dans les secteurs siliceux, parfois associées au genévrier nain (Juniperus sibirica) et, enfin, les saulaies avec de différents types (en particuier, dans l'aire dolomitique prévalent les aspects à prédominance de Salix waldsteiniana, Salix glabra, Salix mielichhoferi, Salix myrsinifolia, Salix hastata, avec Salix glaucosericea, Salix caesia, Salix foetida plus localisés et seulement dans les aires à l'intérieur) d'importante valeur naturelle, presque toujours des expressions pionnières et sub-hygrophiles. Moins connues et étudiées ce sont les formations à Genista radiata qui, après l'abandon des aires fauchées dans le passé, se sont diffusées dans les milieux herbu-rupestres au soleil, en contact avec les bois de Ostrya carpinifolia, et qui en altitude parviennent même à $1900 \mathrm{~m}$. On peut faire égale considération pour les aspects à néflier des rochers (Amelanchier ovalis), que presque toujours on peut associer à des milieux de pinède potentielle et essentiellement azoneaux. Encore à étudier ce sont les formations, toujours dans des milieux ardus et bien au soleil, des substrats volcaniques (en Agordo en particulier) dominées par Juniperus sabina, qui posent aussi d'importants problèmes de syntaxonomie. Si la bruyère haute, à Rhododendron ferrugineum, c'est une formation zonale à la limite supérieure de la forêt, également spectaculaire c'est la bruyère basse à azalée naine - (Loiseleuria procumbens), au faux myrtille (Vaccinium gaultherioides) ou à Empetro-Vaccinietum, parfois associé à des sphaignes (Sphagnum sp.pl.). De toutes les formations forestières, décrites ou souvent seulement citées sans la publication des reliefs, c'est la pessière des alluvions à Petasites paradoxus dans les zones internes qui mérite un approfondissement ainsi que la pinède endalpique qui abrite aussi Pinus cembra. Sans comparaison, pour sa richesse, c'est la sapinière sub-montagneuse de ravin, riche en bois latifoliés nobles en particulier Ulmus glabra, étendue dans la Valle del Grisol et en moindre quantité dans d'autres vallées latérales dans la droite hydrographique du Maé. Ce sont encore les bois d'Acer pseudoplatanus, au début de la Vallée de San Lucano, qui méritent une mention. Ils sont presque purs mais, en altitude, ils sont remplacés par des formations à dominance de Sorbus aucuparia, avec la présence plus ou moins étendue d'Alnus viridis. En ce qui concerne la partie du territoire de Belluno, une synthèse récente sur la végétation, surtout forestière, est relatée en Lasen (2020a), tandis que sur les versants de Trento il y a de nombreuses études, même monographiques de Pedrotti ou de son école (sur les brousses à Pinus mugo, Pinus sylvestris, Abies alba). Par contre ce qui manque, ce sont des références récentes pour l'Alto Adige (l'étude typologique est difficilement rapportable à un tableau phytosociologique). Pour Trento et Bolzano, on peut encore utiliser les manuels, même si destinés aux objectifs d'application de Nature 2000, respectivement Lasen 2006 pour Trento; Lasen \& Wilhalm, 2004 pour Bolzano. Pour la région du Friuli il y a beaucoup de travaux monographiques sur les seules catégories par l'oeuvre de Poldini et son école. 


\section{Systèmes de tutelle et patrimoine UNESCO}

L'absolue qualité de la nature et du paysage des territoires dolomitiques est affirmée par le nombre et l'extension des aires sous tutelle pour de variées raisons: les parcs nationaux et régionaux, les réserves naturelles, les biotopes, les sites du Réseau Nature 2000, les aires de nature sauvage.

Même les Plans Territoriaux de coordination et les PRG de quelques communes contiennent des normes spécifiques pour la tutelle des aires de grande valeur pour la conservation. En 2009, enfin, le 26 juin à Séville, le site sériel « Dolomiti» est entré dans la liste du patrimoine naturel mondial de l'humanité pour les critères VII, le paysage, et VIII, la géologie. Les qualités d'unicité et surtout d'intégrité, requises pour entrer dans cette liste, ont déterminé une sélection des aires core et buffer (regroupées ensuite en 9 systèmes), en excluant, justement, les secteurs les plus exploités au niveau touristique. Ce n'est pas un cas si le 95\% de l'aire comprise dans le site UNESCO rentre en parcs, aires protégées ou sites Nature 2000. Dans le domaine dolomitique se trouvent de différentes aires protégées qui, liées entre elles, constituent le principal réseau écologique du pays entier, digne d'être activé et qui devrait représenter une force de dissuasion contre de futurs projets d'exploitation et de consommation du sol.

Tableau 1. Parcs naturels et autres aires protégées établies dans les Dolomites.

Dans la province de Belluno

Parco Nazionale delle Dolomiti Bellunesi: 31512 hectares

Parco Regionale delle Dolomiti d'Ampezzo: 11200 hectares

Dans la region Friuli Venezia Giulia (Pordenone et Udine)

Parco Regionale delle Dolomiti Friulane: 36950 hectares

Dans la province autonome de Trento

Parco Naturale Adamello-Brenta: 62051 (il rentre aussi dans le réseau de Geoparchi)

Parco Naturale Paneveggio-Pale di San Martino: 19727 hectares

Dans la province autonome de Bolzano-Südtyrol

Parco Naturale delle Tre cime: 111892 hectares

Fanes-Senes-Braies: 25453 hectares

Puez-Odle: 10772 hectares

Sciliar-Catinaccio: 7291 hectares

Les nombres déjà indicatifs se réfèrent au domaine global du parc, indépendamment du fait que les aires soient insérées dans la «core» ou dans la «buffer» du périmètre du site sériel UNESCO et que les classifications officielles au niveau national ne tiennent pas en compte les territoires où l'activité de la chasse est permise.

L'existence d'aires protégées et de toutes façon renommées pour leur grande valeur naturelle, c'est le centre du système sériel qui a obtenu la reconnaissance internationale. Mais, en 
dehors de bien connues différences d'ordre administratif et de gestion entre les provinces autonomes et celles ordinaires (le FVG est autonome mais d'une façon assez différenciée de deux provinces du Trentino Alto Adige), l'essentiel c'est de rappeler, qu'en réalité, les directeurs des parcs naturels dans le périmètre sous la tutelle UNESCO avaient entrepris des formes de collaboration bien avant 2009 et, qu'à présent, une liste de bonnes pratiques, dérivée de variées expériences, est disponible.

Ce «réseau de fait», même où il n'a pas été reconnu et institutionnalisé, représente le point fort de l'un des réseaux fonctionnels où le travail de la Fondazione Dolomiti Unesco s'est articulé.

Avec la coordination des deux provinces du Friuli, il existe une table technique avec un groupe de travail qui, après la fusion, est dénommé Réseau du patrimoine du paysage et des aires protégées.

Ce réseau a produit beaucoup de documents et il suffit d'entrer dans le site de la Fondation pour les visionner en partie. Même s'il est vrai que les aspects concernant la tutelle de la biodiversité et du patrimoine naturel ne sont pas un devoir principal du Réseau, vu qu'il ne rentre pas dans les critères pour lesquels le territoire a obtenu la reconnaissance, on ne peut pas nier que la contribution de la qualité naturelle à la valeur du patrimoine du paysage reste un composant basilaire. Par conséquent, même les indicateurs individualisés et nécessaires pour la surveillance et le monitorage de la gestion du bien ont puisé abondamment aux facteurs qui caractérisent un patrimoine naturel, soit en termes d'espèces-parapluie très significatives et faciles au monitoring, soit sur les types d'habitat qui sont les plus emblématiques et caractérisants du paysage dolomitique.

\section{Pressions et menaces}

Les Dolomites sont une marque trop invitante pour penser que la concentration d'aires protégées et la bonne organisation d'ensemble de la gouvernance et des appareils administratifs, soient suffisants pour garantir une tutelle efficace du patrimoine de la nature. Les faits liés aux grands événements sportifs, le Championnat mondial de ski alpin, en février 2021, et les Olympiades d'hiver en 2026 (Milano-Cortina, mais avec des lieux de compétition même dans les provinces autonomes de Trento et Bolzano) témoignent que, malgré le «soutenable» affiché, des projets plus ou moins destructeurs ont surgit et ils ont causé encore des pertes du sol, le sacrifice de parties de bois, la construction d'oeuvres pour les chantiers, les transports, les remontées, l'approvisionnement hydrique.

Les dossiers présentés par des groupes d'associations environnementalistes, le CAI inclus, montrent l'entité des impacts sur le paysages. Les financements publics attirent beaucoup. Il y a de nombreux projets qui prévoient de nouvelles liaisons et des carrousels, menaçant des aires d'évidente valeur, encore intègres et qui rentrent aussi dans le Réseau Nature 2000. L'opposition des associations et des comités locaux, soutenus par quelques communes, ne peuvent pas s'opposer, semble-t-il, aux forts intérêts en jeu. Les gestions par commissaire, rapides dans l'exécution des procédures pour les autorisations, en réalité, portent à dépasser les obligations concernant le milieu qui sont les seules mesures de tutelle et garantie pour limiter les dommages. La monoculture du ski alpin qui cause des impacts évidents, a déjà contribué à corrompre le paysage, en laissant des signes de dégât et dénaturant l'étique de vivre à la montagne. 
Une conception de développement qui implique une augmentation de consommation et favorise le tourisme de masse est incompatible souvent avec les fragiles équilibres écologiques.

Les faits liés à la pandémie ont fortement limité l'accès aux pistes et mis en évidence, que l'on ne peut pas soutenir dans le moyen et long terme le dessein de viser à de nouvelles remontées, de nouvelles pistes, des canons pour la neige artificielle, des routes de service, des lieux pour la restauration et tout l'induit. Même en relation au changement du climat en action, une rationalisation de ce qui existe, s'annonce comme la seule solution compatible pour la tutelle de la Nature restante. On sait bien que beaucoup de fonds de la vallée dolomitiques ont été occupés par des infrastructures, semblant ainsi à des secteurs de la plaine, et que le réseau écologique en est compromis. Les évènements répétitifs, liés aux précipitations intenses qui causent des éboulements et des alluvions, sont bien sûr naturels, mais certes favoris par des choix qui ont privilégié la réalisation des profits par rapport aux circonspections nécessaires à la tutelle des équilibres écologiques. En général tout le secteur touristique provoque des pressions de différente nature, concentrées dans quelques vallées et sites, tandis que dans d'autres les accès sont inférieurs à l'expectative et au seuil de la capacité accueillante. Les études coordonnées par la Fondazione Dolomiti Unesco ont mis en évidence que le nombre de touristes dans deux lieux symbole tels que le Lac de Braies et les Trois Cimes de Lavaredo dépasse largement la capacité d'accueil du système, avec des milliers de visiteurs pendant les mois d'été.

Dans l'attente des publications officielles à ce propos, on peut trouver des informations sur les sites de la Fondazione: https://www.dolomitiunesco.info/dolomiti-unesco-lo-studio-per-unagestione-degli-accessi-nei-luoghi-piu affollati/ et de la Provincia Autonoma di Bolzano: http://www.provincia.bz.it/news/it/news.asp?news_action=4\&news_article_id $=645521$

L'accès au Lac du Sorapiss est un autre point chaud qui a fait couler beaucoup d'encre et qui reste un thème central de la région dolomitique entière. Il faut limiter le trafic routier privé des automobiles et des motos à travers les cols dolomitiques. Ce n'est pas ici que l'on veut disserter profondément, ni faire une liste des situations critiques sur la réglementation et développement du tourisme dolomitique, mais, partout, il est évident que l'impact sur les dynamiques écologiques est important et il y faut des attentions et des circonspections. A ce propos on pourrait commencer des recherches avec des monitorages continuels et périodiques de quelques variantes, observant les indicateurs bien connus. Par exemple, les groupes des lichens près des cols les plus fréquentés, pour évaluer la pollution atmosphérique, la diffusion d'espèces allochtones, etc.). Mais les Dolomites n'auraient pas la renommée dont elles jouissent, justement, au niveau planétaire, si leur paysage ne fût pas rapportable à une gestion soignée des milieux qui traditionnellement sont le centre de toute économie de la montagne: la sylviculture et l'alpiculture. L'évolution socioéconomique à provoqué l'abandon de vastes aires à prairies à cause de la difficulté d'accès et des coûts. En bonne partie, cela est le résultat des choix politiques. Par exemple, en Alto Adige, la situation est bien différente par rapport aux territoires limitrophes. En plusieurs cas, en effet, le vrai problème c'est l'écoulement du purin et des excréments des animaux, pour répondre aussi à la directive européenne sur les déversements d'eaux sales zootechniques. Beaucoup de cabanes de bergers développent encore leur activité et comptent aussi sur les ressources apportées par les touristes de passage (restauration et vente des produits), d'autres ont été abandonnées, ou en phase de dégradation, ou bien elles ont été restructurées comme refuge pour le maintien du paysage. La vraie émergence n'est pas représentée par les pâturages, mais par les 
prés à faucher toujours plus rares qui, toutefois, en particulier pour la conservation de la biodiversité de la flore et de la végétation, ont un rôle fondamental.

Il reste les prairies alpines primaires avec leurs floraisons spectaculaires, mais elles aussi sont souvent menacées à cause du changement du climat qui favorise la remontée de la limite des espèces ligneuses. La délicatesse de la conservation des biotopes humides se heurte aux exigences pratiques et aux œuvres des chantiers qui, enfin, en déterminent la fragmentation et le déclin. Ce thème a été bien reconnu et il ne manque pas d'exemples édifiants. Pour ce qui concerne la gestion forestière, habituellement les bois en haute altitude sont classés de «protection» et non de «production», mais, la diffusion des routes forestières qui, en réalité, favorisent aussi les accès touristiques et la chasse, n'aide pas les politiques de réelle conservation et, en particulier, l'introduction de quelques parcelles laissées à l'évolution naturelle, sans utilisation, vers le bois vétuste plus proche-naturel. C'est un choix de fondamentale importance stratégique et capable d'élever encore plus la valeur ajoutée de ces territoires. On pourrait aussi réaliser de vrais laboratoires en plein air où l'on pourrait mesurer les effets des changements en action. Nombre de bois en haute altitude ont été dans le passé des pâturages et, aujourd'hui, on observe que la réduction de la charge baisse la pression et elle favorise l'arolle qui était pénalisé par rapport au mélèze. En automne, les étonnantes couleurs des mélèzes et des arolles sont l'un des éléments les plus caractéristiques du paysage dolomitique. Mais, la conservation de beaux bois de mélèzes est en péril sans des interventions appropriées, comme le démontrent les successions de bois de mélèzes et de sapinières que l'on peut souvent observer dans une vaste zone d'altitude et qui ne contribuent pas à améliorer la perception du paysage.

\section{Perspectives et dynamiques évolutives}

Si, faire le point sur l'état des connaissances, même dans une sélection partielle, il signifie de traiter essentiellement du passé, plus proche que lointain, il devient décisif de comprendre la direction du dynamisme naturel et d'imaginer le scénario du paysage dolomitique des décennies à venir. Les observations directes concernent la dernière moitié du siècle et elles ont subi une accélération dans les derniers temps, soit pour les effets du changement climatique, soit pour ceux dérivés des interventions de l'homme qui ont affaibli souvent la résistance de certains écosystèmes. Si, dans une certaine mesure, la fragmentation des phytocénoses et les interventions de l'homme peuvent provoquer une augmentation de la biodiversité (et cela ne signifie pas de qualité d'ensemble), déjà à moyen terme, il y aura des effets négatifs causés par des gestions agro-sylvo-pastorales irrespectueuses des principes de la conservation écologique. Dans certains cas, l'abandon ne cause pas des désastres écologiques, mais il conduit à des phases de transition bien moins spectaculaires et attractives. Les facteurs qui interagissent sont complexes et les situations articulées selon les différentes vallées et zones, mais quelques éléments à caractère général surgit clairement.

La limite de la forêt et des arbustes va grandir, comme prévu, par la suite de l'augmentation des températures moyennes. On peut le prouver par les comparaisons photographiques. Le fait qu'un nombre important d'espèces herbacées soit aujourd'hui repérable à des altitudes jamais observées auparavant, c'est une donnée bien documentée. Parmi les 
graminacées dominantes sur quelques pentes escarpées, exposées au sud, Molinia arundinacea a dépassé $1900 \mathrm{~m}$. et Brachypodium caespitosum va au dessus de $2100 \mathrm{~m}$.

Les études sur les petites vallées nivales des Alpi Feltrine démontrent un recul des espèces de Salicetea herbaceae et une entrée toujours plus grande d'espèces pabulaires de grande valeur écologique. (Tomaselli \& al. 2005; Tomaselli ex verbis). On signale aussi le rapprochement de quelques entités thermophiles, en particulier des orchidées, vers l'aire dolomitique, même si le phénomène n'est pas encore éclatant.

Les événements extrêmes, comme celui de Vaia, comportent des bouleversements écologiques qui font paraître des successions sérielles imprévisibles ou, au moins, bien peu étudiées et jamais signalées auparavant. Des observations au mois de mai 2020 en Alto Agordino ont vérifié, auprès des souches arrachées par la violence du vent, une prolifération de Corydalis capnoides, espèce connue seulement dans une station avec peu d'exemplaires, au point d'être évaluée CR dans la liste rouge provinciale. Même la Lappula squarrosa s'est multipliée en abondance, parvenant à l'altitude de $1700 \mathrm{~m}$. Dans le paysage dolomitique, les espèces allochtones trouvent peu d'opportunité pour leur diffusion, surtout en altitude. Parmi les exceptions il y a Erigeron annuus, espèce envahissante, même en montagne, dans des milieux proche-naturels. Dans les bois humides et non cultivés des fonds de la vallée Impatiens glandulifera est en expansion. La concentration d'entités allochtones est inversement proportionnelle à l'altitude et au niveau du bouleversement. Le long des cours fluviaux, le Piave en particulier, le nombre et la couverture de ces entités augmentent en procédant des sources jusqu'au débouché vers la haute plaine. En extrême synthèse, sans recours à des scores, surtout discutables, il peut être utile de formuler des indications sur la tendance évolutive telles qu'elles apparaissent après les 50 années d'observations sur le champ comme macro-catégorie de l'habitat.

- Milieux némoraux. Dans l'ensemble ils sont les moins critiques, excepté ceux humides dans les fonds de la vallée ou dans des stations où la compétition de Robinia pseudacacia et Ailanthus altissima (cette dernière encore marginale) est forte, à la suite d'utilisations peu adroites. Il ne manque pas d'aires qui bénéficient des dernières décennies de trêve et d'économie et où l'on observe une amélioration d'ensemble au niveau de la qualité de la nature, c'est à dire de rapprochement à des conditions plus voisines à celles de la végétation potentielle. Pour citer un exemple, la récupération du rouvre (Quercus petraea) dans le domaine de orne-ostryetum et la bonne tenue des communautés riches en bois latifoliés nobles (Tilio-Acerion s.1.) dans de milieux de ravin et non seulement. Parmi les conifères, Abies alba et Pinus cembra sont en récupération remarquable.

- Formations à arbustes. Soit celles thermophiles, soit surtout celles tempérées-boréales et subalpines paraissent en progrès appréciable et en bon état de santé. Leur expansion est en partie déterminée par la moindre pression du pâturage et en partie par les effets du changement du climat. La situation de quelques saulaies est plus délicate, soit parce qu'elles sont essentiellement rares, soit parce qu'elles gravitent dans des milieux humides à plus grand péril.

- Formations herbacées. Elles souffrent en général pour de variées raisons, les prés gras à cause de l'abandon ou des engrais excessifs et aussi pour des irrégularités de la gestion. A présent il est difficile d'observer de vastes zones à trisètes en très bonnes conditions. On trouve une meilleure situation pour les arrhenateraies, même si elles ont subi une remarquable réduction de leur aire. Les aires à pâturage où prédominent les aspects à Poion alpi- 
nae, ressentent elles aussi de gestions alternes et rarement optimales. Il y a quelque exception dans les Dolomites d'Ampezzo. Pour des causes naturelles et pour l'absence de gestion, les prairies de type aride-steppique sont en forte diminution, déjà rares pour des raisons historico-climatiques. Elles mériteraient une attention spécifique avec des interventions appropriées. Enfin, il faut souligner que la biodiversité de quelques secteurs est protégée justement par des irrégularités de gestion qui gardent d'élevés niveaux de diversification, ralentissant ainsi la banalisation et la diffusion de communautés nitrophiles.

- Milieux humides. Même s'ils sont souvent recensés et individualisés comme des sites Nature 2000 ou des biotopes, les situations critiques de base, innées dans leur fragilité et leur sensibilité, restent bien présentes. Dans quelque cas ils nécessitent de protections spécifiques pour éviter l'abandon à la libre évolution ou aux effets négatifs causés par la proximité de routes, de pistes, de lignes de débardage. Il faudrait interdire de nouveaux drainages, parfois justifiés pour des raisons de sûreté. Dans d'autres cas, au contraire, il est convenable de les préserver du pâturage qui, au cours des saisons sèches, peut causer des effets délétères. En général, les sources, là où il n'y ait pas d'indications d'espèces rares et tous les milieux humides sont importants pour la biodiversité surtout animale, et donc ils exigent du respect. Pour les miroirs d'eau, en outre, il faut individualiser les mesures appropriées pour ralentir ou empêcher l'eutrophisation qui, néanmoins est un phénomène naturel, en partie. Enfin, on connaît les contrastes entre protectionnistes et hydrauliciens ou forestiers pour la gestion des communautés péri-fluviales. Sans entrer dans les détails de la diatribe, il faut distinguer les situations des cénoses des grèves, conditionnées par les prélèvements et par la réglementation du régime, de celles des milieux riverains plus mûrs qui développent des fonctions écologiques de primaire valeur.

- Les milieux typiquement dolomitiques représentés par les communautés pionnières des parois rocheuse et/ou des éboulis de faille, particulièrement ravissantes dans beaucoup de secteurs, jouissent généralement de bonne santé à l'exception de cas extrêmes ou très localisés, ils ne nécessitent pas d'intervention particulières.

- Pour les prairies primaires d'altitude, habituellement non fauchées et éventuellement exposées au pâturage extensif, les effets du changement du climat peuvent être négatifs. Ces effets se manifestent déjà, en toute évidence dans la réduction, qualitative et quantitative, des phytocénoses des petites vallées nivales.

\section{Remerciements}

Avec Michele Da Pozzo, Filippo Prosser, Marcello Tomaselli, Thomas Wilham et le secrétariat de la Fondation on s'est confrontés sur quelques données avec d'utiles échanges de vue.

Silvana Longiarù a traduit, aidée aussi par sa passion et par la connaissance du territoire.

\section{Bibliographie}

AA.VV. (by Gasparo, D. \& Zappa, L.), 1994: Organismi come bioindicatori ambientali. -Trieste. AA.VV. (by Pirola, A.) 2000: Contributi alla valutazione dell'impatto ambientale con indicatori floristici e vegetazionali. - Archiv. Geobotanico 5(1-2), 1999. 
Argenti, C. \& Lasen, C. 2001: La flora. - P. 210 in: Studi e Ricerche del Parco Nazionale Dolomiti Bellunesi, 3. - Castelfranco Veneto.

— \& - 2004: Lista rossa della flora vascolare della Provincia di Belluno. - Vicenza.

Biondi, E., Blasi, C., Allegrezza, M., Anzellotti, I., Azzella, M. M., Carli, E., Casavecchia, S., Copiz, R., Del Vico, E., Facioni, L., Galdenzi, D., Gasparri, R., Lasen, C., Pesaresi, S., Poldini, L., Sburlino, G., Taffetani, F., Vagge, I., Zitti, S. \& Zivkovic, L. 2014: Plant communities of Italy: The Vegetation Prodrome. - Pl. Biosyst. 148(4): 728-814, https://doi.org/10.1080/11263504.2014.948527

Blasi, C. (ed.): La vegetazione d'Italia, con carta delle serie di vegetazione in scala 1:500.000. Roma.

Boiti, I., Lasen, C. \& Saffaro Boiti, T. 1988: Interessante Aspekte im Refugialgebiet des VenegiaTales. - Sauteria 4: 121-131.

—, — \& - 1989: La vegetazione della Val Venegia. Provincia Autonoma di Trento. - Calliano.

Bonomi, C. \& Buffa, G. 2000: Valutazione della qualità della vegetazione della torbiera delle Viote del Monte Bondone (TN) con lineamenti di corretta gestione ambientale. - Studi Trent. Sc. Nat. 74(1997): 77-97.

Buffa, G., Carpenè, B., Casarotto, N., Da Pozzo, M., Filesi, L., Lasen, C., Marcucci, R., Masin, R., Prosser, F., Tasinazzo, S., Villani, M. \& Zanatta, K. 2016. Lista rossa regionale delle piante vascolari. - Venezia.

Cerabolini, B., Raimondi, B., Cattaneo, M., Preatoni, D. \& Brusa, G. 2007: I caratteri della vegetazione come descrittori della qualità ambientale: un'applicazione cartografica (provincia di Varese, Lombardia). - Inform. Bot. Ital. 39(1): 155-165.

Da Pozzo, M. \& Lasen, C. 2010: Le zone umide di interesse naturalistico nelle Dolomiti d'Ampezzo. - Frammenti 2: 9-46.

Del Favero, R. (by), Abramo, E., Andrich, O., Corona, P., Cassol, M., Lasen, C., Marchetti, M., Carraro, G., Dissegna, M., Giaggio, C., Savio, D. \& Zen, C. 2000: Biodiversità e Indicatori nei tipi forestali del Veneto. - Venezia.

Favero, P. \& Lasen, C. (by) 2016: La Riserva Naturale Orientata di Somadida. Scrigno di biodiversità e luogo di boschi vetusti. - Corpo Forestale dello Stato - Rasai di Seren del Grappa.

Ferrari, C., Pezzi, G. \& Dell'aquila, L. 2000: Diversità e naturalità della vegetazione. Elementi per un'analisi quantitativa integrata. - Inf. Bot. Ital. 32 (suppl. 1): 31-34. Atti XIV Convegno G. Gadio.

Fondazione Dolomiti Unesco 2014: "Linee guida per il paesaggio" convenzione di ricerca tra l'Università degli Studi di Udine, la Provincia di Udine e la Fondazione Dolomiti-DolomitenDolomites-Dolomitis UNESCO nell'ambito dei lavori della Rete funzionale del Patrimonio Paesaggistico e Aree Protette.

Lasen, C. 1982: Vegetazione nivale a Luzula alpino-pilosa nelle Alpi Feltrine. - Studi Trent. Sc. Nat. 59: 31-40, Trento.

- 1989a: Varietà floristica e vegetazionale nel paesaggio dolomitico, una risorsa in pericolo da apprezzare e tutelare. - Pp. 121-131 in: "Dolomiti, risorsa dell'Europa". Atti del convegno di Cortina d'Ampezzo 7-8 aprile 1989.

— 1989b: Note floristiche, ecologiche e fitosociologiche sui salici della Val Venegia (Dolomiti sudoccidentali). - Studi Trent. Sc. Nat. 65: 49-61.

- 2000: Paesaggio vegetale e flora. - Pp. 366-375 in: De Battaglia, F. \& Marisaldi, L. (eds), Enciclopedia delle Dolomiti. - Bologna.

— 2001: Le Dolomiti. - Pp. 68-79 in: AA.VV.: Parchi Montani. - Forli.

— 2004: Sintesi descrittiva e valori vegetazionali. - Pp. 153-176 in: Cason Angelini, E. (ed.), Un Parco per l'uomo. Dieci anni di vita del Parco Nazionale Dolomiti Bellunesi. - Belluno.

- 2006: Habitat Natura 2000 in Trentino. - Trento. 
- 2008: Natura e paesaggi del territorio bellunese. - Pp. 25-139 in: Lasen, C. (ed.), Tesori naturalistici. Viaggio alla scoperta dei paesaggi e della biodiversità, dalla montagna al mare, nelle province di Belluno, Vicenza, Verona, Mantova, Ancona. - Verona.

- 2009: Paesaggio vegetale e valori floristico-vegetazionali in Centro Cadore e Dolomiti di Sinistra Piave. - Pp. 43-70 in: Begotti, Pc. \& Majoni, E. (ed.), Dolomites. - Atti del LXXXVI Congresso della Società Filologica Friulana, Pieve di Cadore 20-09-2009. - Udine.

-2012: Dolomiti. La promozione del bene Unesco. - Pp. 271-276 in: Varotto, M. \& Castiglioni, B. (ed.), Di chi sono le Alpi? Appartenenze politiche, economiche e culturali nel mondo alpino contemporaneo. - Rete Montagna, Università di Padova. - Atti del VI Convegno internazionale di Rete montagna, Agordo 22-24 settembre 2011.

— 2016: Lineamenti vegetazionali. Pp. 244-251 in: Da Pozzo, M., Argenti, C. \& Lasen, C.: Atlante floristico delle Dolomiti d'Ampezzo. Specie notevoli, valori ecologici e fitogeografici. - Cortina d'Ampezzo.

- 2020a: Lineamenti della vegetazione in provincia di Belluno. - Pp. 13-58 in: Padovan, F., Lorenzon, L., Campo, E., Floriani, M., Michelin, L., Brotzu, R., Bizio, E. \& Magnozzi, M.: 1260 funghi della Provincia di Belluno. Atlante fotografico. - Belluno.

- 2020b: La Natura: tra vincoli, interessi contrapposti e opportunità di recupero. - Pp. 23-38 in: Viola, F. (ed.), Cause ed effetti della devastazione nell'area montana triveneta. Giornata di Studi 30 gennaio 2019. - Padova.

— \& Da Pozzo, M. 2011: La flora e la vegetazione. - Pp. 94-145, with redlist pp. 322-327 and bibliography p. 331 in: Alberti, A., Cassol, M., Da Pozzo, M., Lasen, C. \& Siorpaes, C. 2011: Dolomiti d'Ampezzo. Guida alla conoscenza delle meraviglie naturali di Cortina. - PCortina d'Ampezzo.

— \& - 2017: Carex maritima Gunnerus (Cyperaceae) rara specie artico-alpina scoperta sulle Dolomiti d'Ampezzo. - Frammenti 7: 95-99.

— \& Pignatti, S. 2002: Spruce forests of the Dolomites and related areas of the South-Eastern Alps. Ecology, management and syntaxonomy. - Razprave IV. Razreda SAZU, XLIII-3: 267-282. Ljubljana.

— \& Scariot, A. 2007: Le macrofite. - Pp. 155-186 in: AA.VV. (by ARPAV, Belluno), 2007: Studio della qualità delle acque dei piccoli laghi alpini. - Venezia.

— \& Wilhalm, T. 2004: Natura 2000. Habitat in Alto Adige. - Bolzano.

—, Pignatti, E. \& Pignatti, S. 1992: Le comunità a Matteuccia struthiopteris nelle Dolomiti sudoccidentali. - Ann. Bot. (Roma) 48 suppl. 7(1990): 155-162.

—, —, — \& Scopel, A. 1977: Guida Botanica delle Dolomiti di Feltre e di Belluno. - Calliano (TN).

Lippert, W., Müller, N., Rossel, S., Schauer, T. \& Vetter, G. 1995: Der Tagliamento- Flußmorphologie und Auenvegetation der größten Wildflußlandschaft in den Alpen. - Verein zum Schutz der Bergwelt e.V. München: 11-70.

Marchiori, S., Rizzo, F., Medagli, P., Albano, A. \& Farenga, T. 2000: Indicatori e metodi per la valutazione della qualità ambientale nella pianificazione territoriale nel comune di Vergole (Lecce). - Inform. Bot. Ital. 32 suppl. 1: 21-25.

Mazzocchi, F., Castorina, M. \& De Mei, M. 1999: Rappresentazione sintetica della qualità ambientale attraverso l'integrazione di indici floristici e vegetazionali: il caso di Macchia Grande di Ponte Galeria (Roma). - Roma.

Melzer, A. 1999: Aquatic macrophytes as tools for lake management. - Hydrobiologia 395/396: 181-190. Mucina, L., Bültmann, H., Dierßen, K., Theurillat, J. P., Raus, T., Čarni, A., Šumberová, K., Willner, W., Dengler, J., Gavillán Garcia, L., Chytrỳ, M., Háyek, M., Di Pietro, R., Iakuschenko, D., Pallas, J., Daniềls, F. J. A., Bergmeier, E., Santos Guerra, A., Ermakov, N., Valachovič, M., Schaminée, J. H. J., Lysenko, T., Didukh, Y. P., Pignatti, S., Rodwell, J. S., Capelo, J., Weber, H. E., Solomeshch, A., Dimopoulos, P., Aguiar, C., Hennekens, S. M. \& Tichỳ, L. 2016: Vegetation of Europe: hierarchical floristic classification system of vascular plant, bryophyte, 
lichen and algal communities. - Appl. Veg. Sci. 19 supplement 1: 3-265. http://dx.doi.org/10.1111/avsc. 12257

Pignatti, E. \& Pignatti, S. 1960: Un'associazione rupestre endemica nelle Dolomiti (Phyteumateto Asplenietum seelosii) all'estremo orientale della sua area. - N. Giorn. Bot. Ital., n.s., 66 (4, 1959): $1-5$.

— \& - 1974: Guida botanica alla Val di Fassa. - Inform. Bot. Ital. 6: 116-130.

— \& - 1978: Über die Campanula morettiana-Vegetation in den Dolomiten. - Mittl. Ostalp.-din. Ges. f. Vegetskde. 14: 279 - 291.

— \& - 1981: Su alcune nuove associazioni vegetali delle Dolomiti. - Giorn. Bot. Ital. 115: 138-139.

— \& - 1983: Danthonio - Nardetum eine neue Bürstlinggesellschaft aus dem Südrand der Ostalpen. Contributions, IV, 1 - 2 - Section of Biological and Medical Sciences. - Skopje.

— \& - 1984a: La vegetazione delle Vette di Feltre al di sopra del limite degli alberi. - Studia Geobot. 3(1983): 7-47. Trieste.

— \& - 1984b. Zur Syntaxonomie der Kalkschuttgesellschaften der südlichen Ostalpen. - Acta Bot. Croatica 43: 243-255.

— \& - 1985: Das Caricetum rupestris, eine neue Assoziation der Südtiroler Dolomiten. - Tuexenia 5: $175-179$.

— \& - 1988a: Introduzione al paesaggio vegetale delle Dolomiti. - Studi Trent. Sci. Nat. 64(suppl.): 13-26.

— \& - 1988b: Zur syntaxonomie der Trifolium nivale-reichen Almwiesen in den südlichen Ostalpen. Sauteria 4: 55 - 69 .

— \& - 1995: Lista delle unità vegetazionali delle Dolomiti. - Atti dei Convegni Lincei 115, XI Giornata dell'Ambiente: La vegetazione Italiana (Roma, 5 giugno 1993): 175-188. - Roma.

— \& - 2003: Le Dolomiti come modello di lettura del paesaggio montano e delle sue trasformazioni. - Inf. Bot. Ital. 35(2): 371-372. - Atti del Convegno: Il patrimonio vegetale delle Montagne. Valori e problemi delle montagne europee ed extraeuropee, metodi di studio. Bergamo 31 maggio-1 giugno 2002.

— \& - 2014-2017: Plant life of the Dolomites, 1-3. - Heidelberg, New York, Dordrecht, London.

_, - , Pietrosanti, S. \& Paglia, S. 1996: La flora delle Dolomiti come archivio informatizzato. - Atti $24^{\circ}$ Simposio Soc. Estalpino-dinarica di Fitosociologia. Rovereto 2-6 luglio 1995. - Ann. Mus. Civ. Rovereto, suppl., 11 (1995): 27-43.

Pignatti, S. 1981: Carta dei complessi di vegetazione di Cortina d'Ampezzo. - Roma. — 1998 (1995): Die Dolomiten-Vegetation als System. - Acta Bot. Croat. 54: 89-96.

Poldini, L. (with Oriolo, G. \& Vidali, M.) 2002: Nuovo Atlante corologico delle piante vascolari nel Friuli Venezia Giulia. - Trieste.

— \& Pertot, M. 1991: Criteri di indicizzazione del valore naturalistico sull'esempio del Carso Triestino - Goriziano. - Inform. Bot. Ital. 21(1-3): 133-151.

Prosser, F. 2001: Lista Rossa della Flora del Trentino. Pteridofite e Fanerogame. - Rovereto.

— \& Bertolli, A. 2008: A new species of Gentiana sect. Calathianae (Gentianaceae) from the Brenta Group. European Alps, Italy. - Willdenowia 38: 423-431.

—, 一, Festi, F. \& Perazza, G. 2019: Flora del Trentino. - ERovereto.

Rodaro, P., Scotton, M., Ziliotto, U. \& Cerantola, L. 1998: Studio vegetazionale e agronomico dei pascoli di Gotres (Cortina d'Ampezzo, BL, Italia). - Doc. Phytosoc. 18: 73-95.

Rossi, G., Tomaselli, M. \& Gualmini, M. 2000: Messa a punto metodologica sul problema dell'indicizzazione del valore naturalistico delle comunità vegetali. - Arch. Geobot. 5(1-2), 1999: 129133.

Scotton, M., Rodaro, P. \& Ziliotto, U. 1996: Analisi fitosociologica e valutazione agronomica di pascoli subalpini (Malga Padeon, Cortina d'Ampezzo, NE Italia). - Studia Geobot. 15: 51-72. 
Tomaselli, M. \& Gualmini, M. 2000: Indicizzazione del valore naturalistico dei pascoli di altitudine nel Parco Regionale dell'Alto Appennino Modenese (Italia settentrionale). - Arch. Geobot. 5(1), 1999: 135-144.

—, Lasen, C., Argenti, C., Gualmini, M., Petraglia, A. \& Nascimbene, J. 2006: Studio Geobotanico di due Biotopi del Parco Naturale Regionale delle Dolomiti d'Ampezzo (Belluno - Italia Nordorientale). - Gredleriana 6: 9-30.

—, — \& Boiti, I. 1989: Zusammenhänge zwischen Vegetation und Oberflächen-karstformen in einigen Gletscherkare der italienischen Dolomiten. - Atti del simposio della Società Estalpinodinarica di fitosociologia, Feltre 29/6-3/7 1988: 127-133.

—, Petraglia, A. \& Lasen, C. 2005: Flora briologica e vegetazione delle vallette nivali nelle Vette di Feltre (Parco Nazionale Dolomiti Bellunesi, Italia settentrionale). - Gortania 26: 111-136.

Viola, F., Gianolla, P., Cassol, M. \& Lasen, C. 2005: Perché le Dolomiti meritano molto di più. Per entrare a pieno titolo nella World Natural Heritage List. - Parchi 46: 50-77.

Wilhalm, T., Niklfeld, H. \& Gutermann, W. 2006: Katalog der Gefäßpflanzen Südtirols. - Ver. des Naturmuseums Südtirols 3: 1-216.

Cet article comprend deux annexes: annexe 1, imprimée, avec les plaques photographiques de certains taxons mentionnés dans le texte; annexe 2, en numérique (ESF1), avec la liste des taxons et syntaxons cites dans le texte et dans les legendes des plaques photographiques.

Adresse de l'auteur:

Cesare Lasen,

Via Mutten 27, I-32032 Arson di Feltre, Belluno, Italie. Email: cesarelasen@gmail.com 


\section{PREMIERE ANNEXE - PLAQUES PHOTOGRAPHIQUES}


Plaque 1: a) Le paysage dolomitique est caractérisé par de vastes couvertures détritiques. Typique des stations enneigées de haute altitude, c'est le Papaver rhaeticum, espèce de l'association Papaveretum rhaetici; b) Leontopodium nivale subsp. alpinum est considéré le symbole de la montagne et autrefois il a été l'objet de cueillettes dans le seul but commercial qui ont réduit l'abondance de sa population; c) Aspects de végétation subalpine en Val Canali où l'on distingue des parcelles de brousses à pin couché qui colonisent les milieux détritiques et où, par la suite, s'installe le mélèze, en contribuant ainsi à caractériser les couleurs du paysage autumnal; d) Pendant la saison printanière les floraisons du narcisse, Narcissus radiiflorus, intéressent les prés de montagne frais et pas trop engraissés. A la suite du changement climatique, le niveau d'altitude va s'éléver et, ici sur le Mont Castello (Vette Feltrine), les prés arrivent jusqu'à plus de 1800 mètres d'altitude; e) Corydalis capnoides; f) Le 29 octobre 2018 une tempête de vent et de pluie torrentielle, dénommée Vaia, a abattu de vastes parties de forêt en causant des dommages jamais observés dans la région dolomitique. Les dynamiques qui s'en succèdent ouvrent des séries inconnues. Sur les substrats du haut Agordino s'est vérifiée une explosion d'une espèce considérée très rare auparavant, la Corydalis capnoides. Elle a trouvé un micro-habitat idéal à la base des souches des arbres écroulés. 


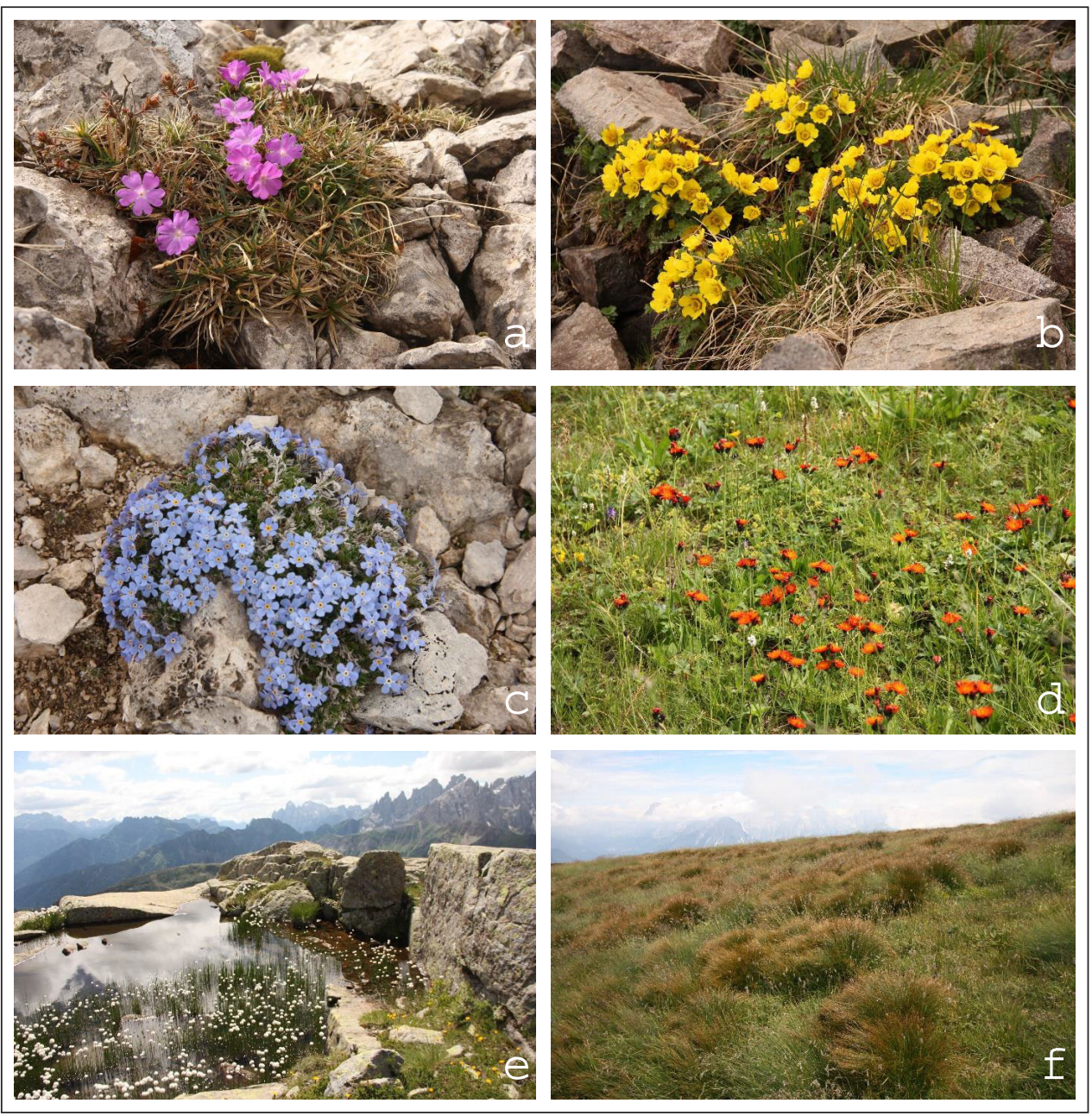

Plaque 2: a) Primula tyrolensis c'est l'un des endémismes le plus répandu du territoire dolomitique, en caractérisant les stations rocheuses fraîches et plutôt humides. Ici avec Carex firma qui est l'espèce colonisatrice prédominante sur les détritus et dans les prairies primaires sur sol primitif (Gentiano terglouensis-Caricetum firmae); b) Geum reptans caractérise la végétation des détritus siliceux et parfois même de ceux carbonatés terrigènes; c) Parmi les espèces les plus spectaculaires des rochers de haute altitude, on remarque Eritrichium nanum, ici sur détritus carbonaté, mais répandu aussi sur des substrates siliceux; d) La gestion pour la conservation des prairies alpines représente l'un des problèmes le plus urgents et de complexe solution. Les zones fauchées sont de plus en plus rares. Ici un aspect où l'on remarque une population de Hieracium auriantiacum; e) Les stations d'altitude élévée à substrat siliceux (ici porphyre) offrent souvent des milieux humides avec des sources, tourbières, mares, et petits lacs. Ici un aspect avec Eriophoretum scheuchzeri au-dessus du lac de Cavia; f) La végétation des prairies alpines des substrats acides (même si dérivant de sols d'origine carbonaté comme ici sur les Vette Feltrine, arête du Mont Vallazza) va rapidement s'évoluer et sur les crêtes et les zones venteuses s'impose une formation à dominance de Juncus trifidus. 


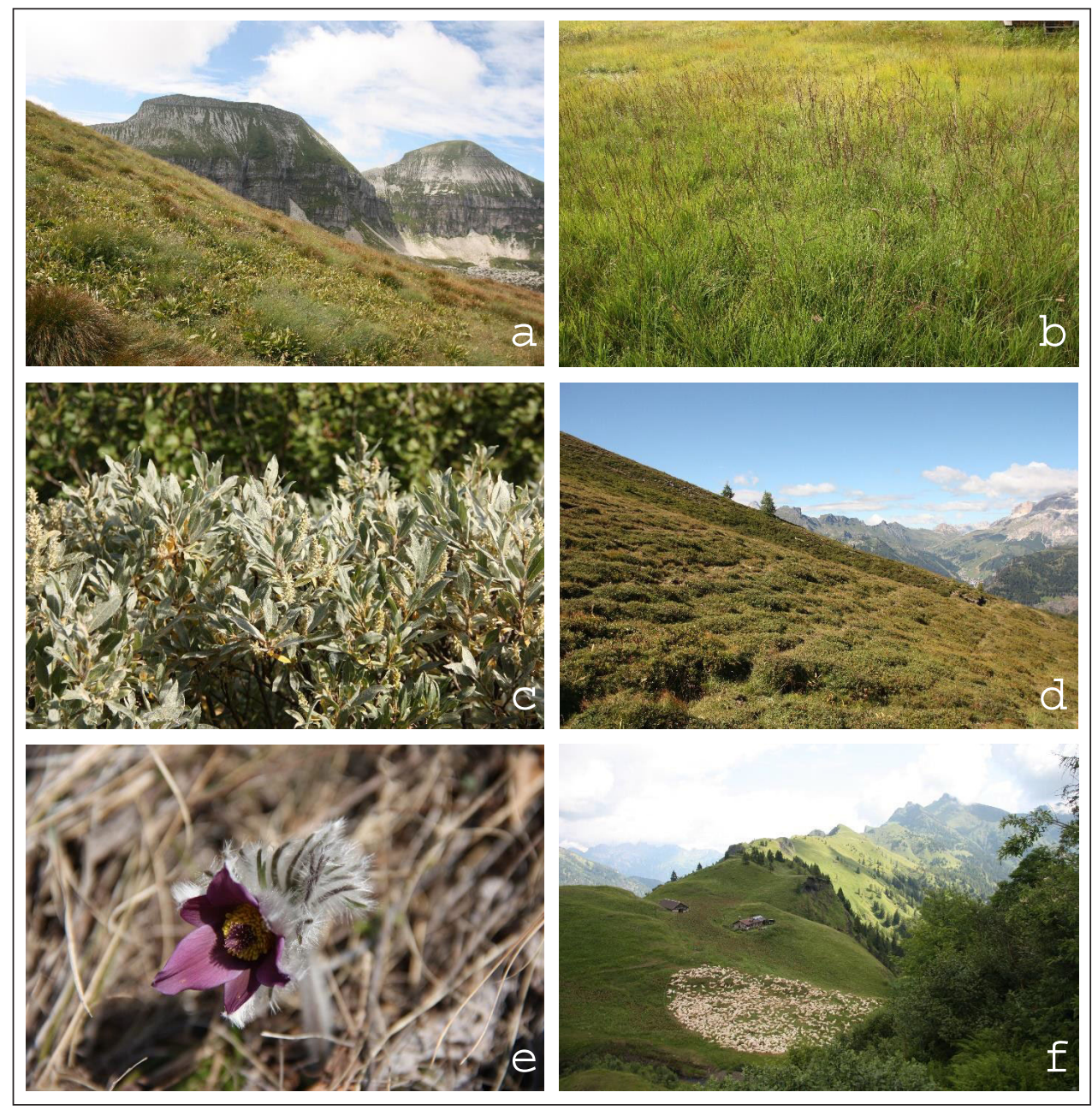

Plaque 3: a) Voilà un autre aspect des versants nord des Vette avec des prairies carbonatées (série Rosso Ammonitico) et des sols acides avec aspects à Gentiana punctata et Juncus trifidus; b) Les zones humides sont toujours d'un grand intérêt pour la conservation. Ici, près de Forcelle Aurine, de moins en moins communes, on trouve des molinaies dans un biotope où l'on remarque aussi des aspects à Rhynchospora alba et Drosera rotundifolia; c) Parmi les espèces les plus importantes genres des territoires alpins, le genre Salix est l'un de plus caractérisant et intéressant. Salix glaucosericea, ici en floraison avec des chatons féminins, est rare dans le domaine dolomitique où il parvient à la partie plus orientale de sa distribution; d) En correspondance des substrats volcaniques ou carbonatés-terrigènes acidifiés aussi, l'abandon du pâturage a favorisé la reprise de la bruyère basse avec de différentes espèces de Vaccinium, Loiseleuria procumbens, Empetrum hermaphroditum et de nombreux lichens et bryophytes; e) Pulsatilla montana est une espèce assez rare et thermo-xérophile qui fleurit bien précocement sur les versants plus à l'extérieur du territoire considéré. Ici sur le Mont Avena dans le Feltrino; f) La gestion des ressources naturelles du territoire dolomitique est devenu un problème sérieux. Le pâturage, pratiqué depuis des siècles avec une surveillance et une alternance correctes, ne représente pas toujours la solution idéale pour la tutelle de la biodiversité. Ici un troupeau de moutons à Campigat avec l'arête de la Paladada. 


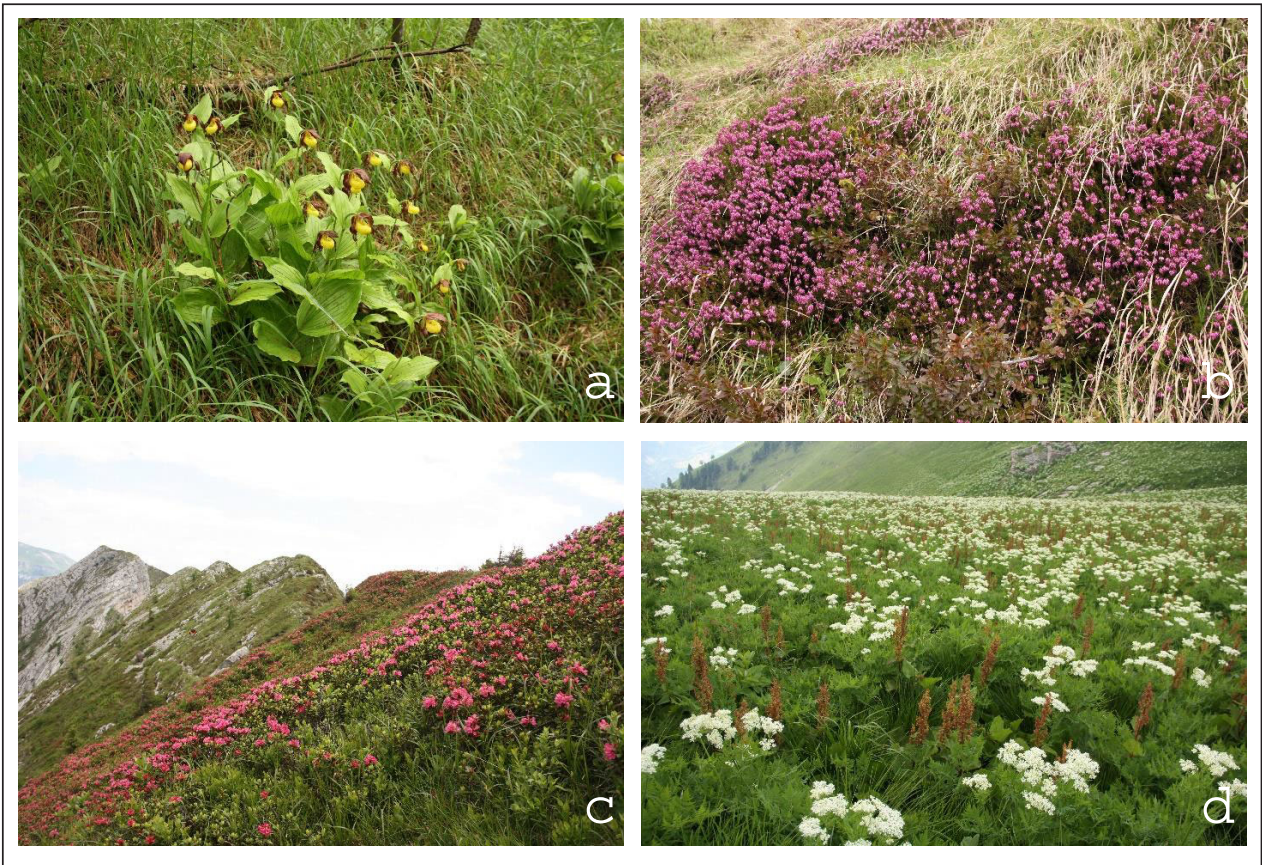

Plaque 4: a) Même si l'espèce est insérée dans l'Annexe II de la directive Habitat 92/43 et sous la tutelle de l'UE, le Cypripedium calceolus est bien représenté dans quelques secteurs du domaine dolomitique et préalpin; b) Beaucoup de territoires dolomitiques sur des versants escarpés des substrats carbonatés sont caractérisés par des formations compétitives sur des sols à humidité alternante, exposés à ruissellement alterné à des périodes seches. Ici une spectaculaire floraison de Erica carnea avec Rhododendron hirsutum, espèce guide; c) Tandis que le Rhododendron hirsutum est exclusif des substrats carbonatés, le Rhododendron ferrugineum aime ceux siliceux, mais il forme de vastes populations aussi sur les substrats carbonatés acidifiés. Ici un aspect de Rhododendron ferrugineum sur l'arête du Mont Coppolo; d) Dans les paysages dolomitiques et préalpins aussi, la présence des cabanes de bergers et le pâturage des bovins et des ovins ont déterminé les communautés végétales dominantes dont l'effet dure longtemps, même après l'abandon. Ici une rare et vaste formation de Myrrhis odorata près de la Vallazza, l'élévation la plus occidentale des Vette Feltrine. 


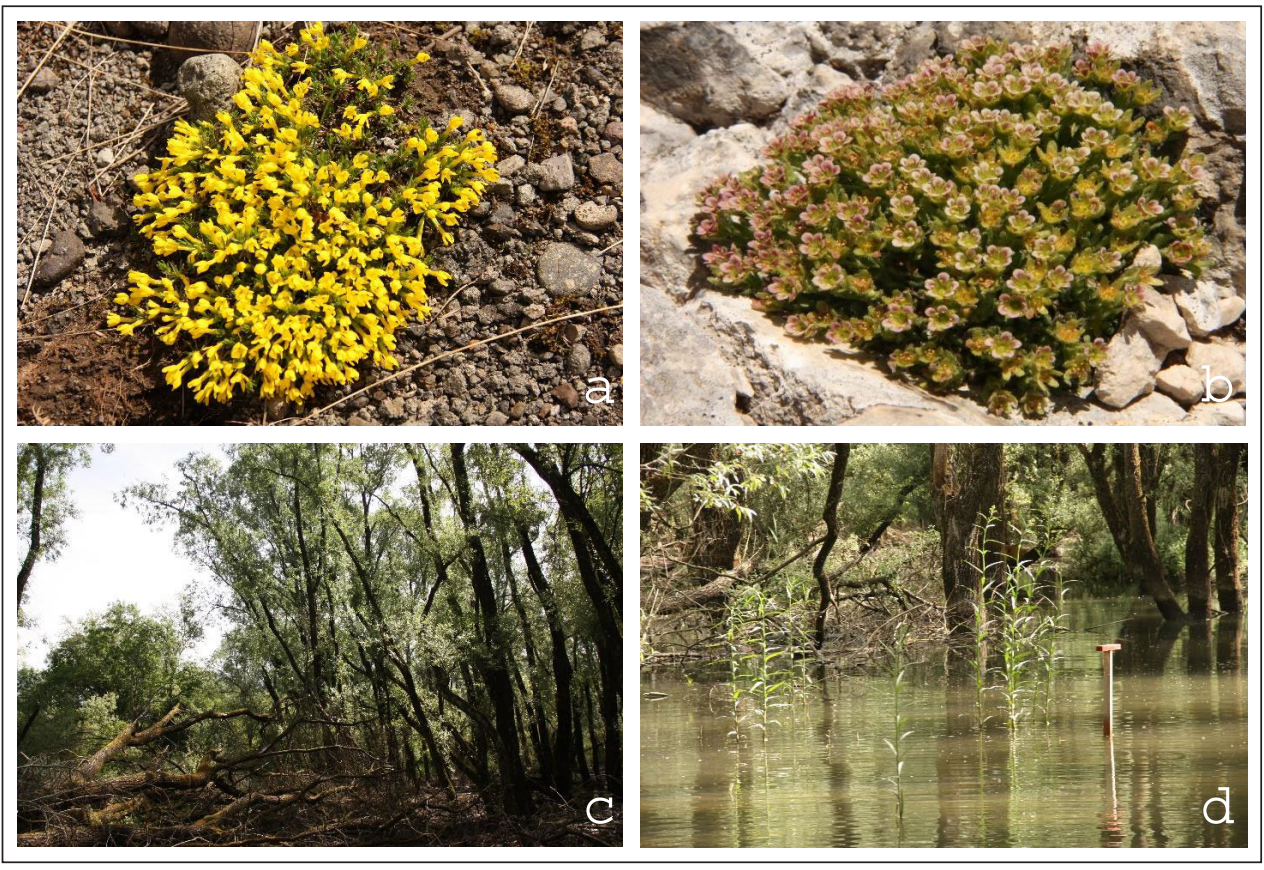

Plaque 5: a) Les substrats volcaniques du haut Agordino (ici la chaîne du Padon) sont vraiment intéressants pour la flore. Ici une floraison de Vitaliana primulaeflora. Aux alentours se trouve la seule station connue dans le territoire de Belluno de Dianthus glacialis; b) Saxifraga facchinii est l'un des endémismes dolomitiques le plus circonscrit. Elle végète seulement sur les crêtes et les parois rocheuses les plus élévées, en corrélation peut-être avec des sites épargnés par les glaciations du quaternaire; c) Aux marges du territoire dolomitique, sur les rives du lac artificiel de Santa Croce, s'est développé un bois ripicole à Salix alba d'une valeur naturelle extraordinaire qui offre surtout des habitats spéciaux pour de différentes espèces animales. Il abrite aussi une nombreuse population du rare Senecio paludosus, ici au début de sa floraison. L'importance du bois mort et des habitats forestiers intacts est ici particulièrement didactique et significative; d) Senecio paludosus. 
Lasen, C.: Biodiversité végétale, valeurs naturelles et sauvegarde du paysage dans le domaine dolomitique. - Fl. Medit. 31 (Special Issue): 521-544. 2022. - ISSN: 1120-4052 printed, 2240-4538 online.

Electronic Supplementary File 1. https://doi.org/10.7320/FIMedit31SI.521.1

Version of Record published online on 6 January 2022

DEUXIEME ANNEXE - LISTE DES TAXONS ET SYNTAXONS CITES DANS LE TEXTE ET DANS LES LEGENDES DES PLAQUES PHOTOGRAPHIQUES

\section{Liste des taxons}

Abies alba Mill.

Acer platanoides L.

Acer pseudoplatanus L.

Ailanthus altissima (Mill.) Swingle

Alchemilla L.

Alnus glutinosa (L.) Gaertn.

Alnus viridis (Chaix) DC.

Amelanchier ovalis Medik.

Asplenium seelosii Leyb.

Betula pendula Roth

Brachypodium caespitosum (Host) Roem. et Schult.

Campanula morettiana Rchb.

Carex bicolor All.

Carex firma Host

Carex maritima Gunnerus

Carex microglochin Wahlenb.

Carpinus betulus L.

Castanea sativa Mill.

Corydalis capnoides (L.) Pers.

Corylus avellana L.

Cypripedium calceolus L.

Dianthus glacialis Haenke

Draba dolomitica Buttler

Drosera rotundifolia L.

Empetrum hermaphroditum Hagerup

Erica carnea L.

Erigeron annuus (L.) Desf.

Eritrichium nanum (L.) Schrad. ex Gaudin 
Fagus sylvatica L.

Festuca austrodolomitica Pils \& Prosser

Fraxinus excelsior L.

Fraxinus ornus L.

Genista radiata (L.) Scop.

Gentiana brentae Prosser \& Bertolli

Gentiana punctata L.

Geum reptans L.

Hieracium L.

Hieracium aurantiacum L.

Impatiens glandulifera Royle

Juncus arcticus Willd.

Juncus trifidus L.

Juncus triglumis L.

Juniperus sabina L.

Juniperus sibirica Burgsd.

Kobresia simpliciuscula (Wahlenb.) Mack.

Lappula squarrosa (Retz.) Dumort.

Larix decidua Mill.

Leontopodium nivale (Ten.) Huet ex Hand.-Mazz. subsp. alpinum (Cass.) Greuter

Loiseleuria procumbens (L.) Desv.

Luzula alpino-pilosa (Chaix) Breistr.

Matteuccia struthiopteris (L.) Tod.

Molinia arundinacea Schrank

Myrrhis odorata (L.) Scop.

Narcissus radiiflorus Salisb.

Nigritella buschmanniae Teppner \& Ster

Ostrya carpinifolia Scop.

Papaver rhaeticum Leresche

Petasites paradoxus (Retz.) Baumg.

Physoplexis comosa (L.) Schur

Picea excelsa (Lam.) Link

Pinus cembra L.

Pinus mugo Turra

Pinus nigra J.F. Arnold

Pinus sylvestris L.

Polystichum lonchitis (L.) Roth 
Populus L.

Populus tremula L.

Primula tyrolensis Schott

Pulsatilla montana (Hoppe) Rchb.

Quercus petraea (Matt.) Liebl.

Quercus pubescens Willd.)

Quercus robur L.

Rhizobotrya alpina Tausch

Rhododendron ferrugineum L.

Rhododendron hirsutum L.

Rhynchospora alba (L.) Vahl

Robinia pseudacacia L.

Rubus L.

Salix L.

Salix alba L.

Salix caesia Vill.

Salix foetida Schleich.

Salix glabra Scop.

Salix glaucosericea Flod.

Salix hastata L.

Salix mielichhoferi Saut.

Salix myrsinifolia Salisb.

Salix waldsteiniana Willd.

Saxifraga depressa Sternb.

Saxifraga facchinii Koch

Sempervivum dolomiticum Facchini

Senecio paludosus L.

Sorbus aucuparia L.

Sphagnum L.

Taraxacum F.H. Wigg.

Taxus baccata L.

Tilia cordata Mill.

Tilia platyphyllos Scop.

Trifolium pratense L. subsp. nivale (Koch) Ces.

Ulmus glabra Huds.

Vaccinium L.

Vaccinium gaultherioides Bigelow

Vitaliana primulaeflora Bertol. 


\section{Liste des syntaxons}

Abieti-Fagetum auct.

Abieti-Piceenion Br.-Bl. in Br.-B1. et al. 1939

Alnetum viridis $\mathrm{Br}-\mathrm{Bl} .1918$

Aremonio-Fagion (Horvat 1950) Borhidi in Török et al. 1989

Betulo pendulae-Populetalia tremulae Rivas-Mart. et M. Costa 1998

Calamagrostio variae-Abietenion (Horvat 1962) Exner et Willner 2007

Campanuletum morettianae Pignatti E. et S. 1978

Caricetum rupestris Pignatti E. et S. 1985

Caricion maritimae Br.-Bl. in Volk 1940 nom. mut. propos.

Carpinion orientalis Horvat 1958

Chelidonio-Robinietalia Jurko ex Hadac et Sofron 1980

Danthonio-Nardetum Pignatti E. et S. 1986

Empetro-Vaccinietum Br.-Bl. in Br.-Bl. et Jenny 1926

Erico carneae-Pinion sylvestris Br.-Bl. in Br.-Bl., Sissingh et J. Vlieger 1939

Erico-Fraxinion orni Horvat 1959 nom. invers. propos

Eriophoretum scheuchzeri Rübel 1911

Gentiano terglouensis-Caricetum firmae T. Wraber 1970

Knautio-Trifolietum Pignatti E. et S. 1988

Larici-Pinetum cembrae [sensu Ellemberg]

Luzulo-Fagion Lohmeyer et Tüxen in Tüxen 1954

Ostryo-Carpinenion orientalis Poldini 1982

Quercion pubescenti-petraeae Br.-Bl. 1932 nom. mut.

Papaveretum rhaetici Wikus 1959

Poion alpinae Gams ex Oberdorfer 1950

Rhodothamno chamaecisti-Laricetum H. Mayer 1984

Tilio-Acerion Klika 1955 